\title{
Complexity of paths, trails and circuits in arc-colored digraphs
}

\author{
Laurent Gourvès $^{1,2}$, Adria Lyra ${ }^{3,4}$, Carlos Martinhon ${ }^{3 \star}$, and Jérôme Monnot ${ }^{1,2}$ \\ 1. CNRS, FRE 3234, F-75775 Paris, France \\ 2. Université de Paris-Dauphine, LAMSADE, F-75775 Paris, France \\ 3. Fluminense Federal University, Inst. of Comp., Niterói, RJ, 24210-240, Brazil \\ 4. Fed. Center of Techn. Educ. Celso S. Fonseca, CEFET/RJ, 26041-271, Brazil \\ \{laurent.gourves, monnot\}@lamsade.dauphine.fr, alyra@ic.uff.br, mart@dcc.ic.uff.br
}

\begin{abstract}
We deal with different algorithmic questions regarding properly arc-colored $s-t$ paths, trails and circuits in arc-colored digraphs. Given an arc-colored digraph $D^{c}$ with $c \geq 2$ colors, we show that the problem of maximizing the number of arc disjoint properly arc-colored $s$ - $t$ trails can be solved in polynomial time. Surprisingly, we prove that the determination of one properly arc-colored $s$ - $t$ path is NP-complete even for planar digraphs containing no properly arc-colored circuits and $c=\Omega(n)$, where $n$ denotes the number of vertices in $D^{c}$. If the digraph is an arc-colored tournament, we show that deciding whether it contains a properly arc-colored circuit passing through a given vertex $x$ (resp., properly arc-colored Hamiltonian $s$ - $t$ path) is NP-complete, even if $c=2$. As a consequence, we solve a weak version of an open problem posed in Gutin et. al. [17].

Keywords: Arc-colored digraphs, Properly arc-colored paths/trails and circuits, Hamiltonian directed path, arc-colored tournaments, Polynomial algorithms, NP-completeness.
\end{abstract}

\section{Introduction, Notation and Terminology}

In the last few years a great number of applications has been modelled as problems in edge-colored graphs $[3,5]$. For instance, problems in molecular biology correspond to extracting Hamiltonian or Eulerian paths or cycles colored in specified pattern $[21,22,10]$, transportation and connectivity problems where reload costs are associated to pair of colors at adjacent edges [13,15], social sciences [9], VLSI optimization [19] among others. In this paper, we are specially concerned (from an algorithmic perspective) with different questions regarding properly arc-colored $s$ - $t$ paths, trails and circuits on arc-colored digraphs.

Given a (not necessarily edge-colored) graph $G=(V, E)$, a trail between $s$ and $t$ in $G$ (called $s$ - $t$ trail) is a sequence $\rho=\left(v_{0}, e_{0}, v_{1}, e_{1}, \ldots, e_{k}, v_{k+1}\right)$ where $v_{0}=s, v_{k+1}=t$ and $e_{i}=v_{i} v_{i+1}$ for $i=0, \ldots, k$ and $e_{i} \neq e_{j}$ for $i \neq j$. A path between $s$ and $t$ in $G$ (called $s$ - $t$ path) is a trail $\rho=\left(v_{0}, e_{0}, v_{1}, e_{1}, \ldots, e_{k}, v_{k+1}\right)$

* Sponsored by FAPERJ and CNPq. 
between $s$ and $t$ where $v_{i} \neq v_{j}$ for $i \neq j$. To extend the definitions above for digraphs we just change edges $e_{i}=v_{i} v_{i+1}$ by arcs (or oriented edges) $e_{i}=$ $\boldsymbol{v}_{\boldsymbol{i}} \boldsymbol{v}_{\boldsymbol{i}+\mathbf{1}}$. In this case, $s$ - $t$ trails (resp., $s$ - $t$ paths) are called directed $s$ - $t$ trails (resp., directed $s$-t paths).

Let $I_{c}=\{1, \ldots, c\}$ be a given set of colors $(c \geq 2)$. In this work, $D^{c}$ denotes a digraph whose arcs have a color in $I_{c}$, with no loops and parallel arcs linking the same pair of vertices. The vertex and arc sets of $D^{c}$ are denoted by $V\left(D^{c}\right)$ and $A\left(D^{c}\right)$, respectively. For a given color $i, A^{i}\left(D^{c}\right)$ denotes the set of arcs of $D^{c}$ colored by $i$. Given $D^{c}$ and two vertices $u, v \in V\left(D^{c}\right)$, we denote by $\boldsymbol{u} \boldsymbol{v}$ an $\operatorname{arc}$ of $A\left(D^{c}\right)$ and its color by $c(\boldsymbol{u} \boldsymbol{v})$. In addition, we define $N_{D^{c}}^{+}(x)=\{y \in$ $\left.V\left(D^{c}\right): \boldsymbol{x} \boldsymbol{y} \in A\left(D^{c}\right)\right\}$ the out-neighborhood of $x$ in $D^{c}\left(d_{D^{c}}^{+}(x)=\left|N_{D^{c}}^{+}(x)\right|\right.$ is the out-degree of $x$ in $\left.D^{c}\right), N_{D^{c}}^{-}(x)=\left\{y \in V\left(D^{c}\right): \boldsymbol{y} \boldsymbol{x} \in A\left(D^{c}\right)\right\}$ the inneighborhood of $x$ in $D^{c}\left(d_{D^{c}}^{-}(x)=\left|N_{D^{c}}^{-}(x)\right|\right.$ is the in-degree of $x$ in $\left.D^{c}\right)$ and $N_{D^{c}}(x)=N_{D^{c}}^{+}(x) \cup N_{D^{c}}^{-}(x)$ the neighborhood of $x \in V\left(D^{c}\right)$. We say that, $T^{c}$ defines an arc-colored tournament if it is obtained from a non-oriented complete edge-colored graph $K^{c}$ by choosing an arbitrary direction for each colored edge of $K^{c}$.

From now on, we write PAC instead of properly arc-colored. A PAC path (resp., PAC trail) is a directed path (resp., trail) such that any two successive arcs have different colors. A PAC path or trail in $D^{c}$ is closed if its end-vertices coincide and its first and last arcs differ in color. They are also refereed, respectively, as PAC circuits and directed PAC closed trails. The length of a directed trail, path, closed trail or circuit is the number of its arcs. Here, we only deal with PAC paths of length greater or equal than 2 .

\subsection{Some Related work}

Problems regarding properly edge-colored paths, trails and cycles (or PEC paths, trails and cycles, for short) in $c$-edge-colored (undirected) graphs have been widely studied from a graph theory and algorithmic point of views (see [3, 1, 24], the book [5] and the recent survey [18]). For instance, in [23], the author gives polynomial algorithms for several problems, including the determination of a PEC $s$-t path (if one exists). More recently, the authors in [1] introduced the notion of trail-path graph. Using this concept, they extend Szeider's Algorithm to deal with PEC $s$ - $t$ trails and they propose a polynomial algorithm for the determination of a PEC $s$ - $t$ trail. A polynomial time characterization of $c$-edge-colored graphs containing PEC cycles was first presented by Yeo [24] and generalized in [1] for PEC closed trails.

When dealing with PEC paths or trails with additional constraints in $c$-edgecolored graphs, the results are less optimistic. For example, it is well known that deciding whether a general 2-edge-colored graph (colored in blue and red) contains a PEC Hamiltonian cycle, a PEC Hamiltonian $s$-t path, or a PEC cycle passing through a prescribed pair of vertices are NP-complete problems [5]. Basically, the idea is to start from the proof of NP-completeness of these problems in uncolored digraphs and to use Häggkvist's transformation which consists in replacing each arc $e=\boldsymbol{x} \boldsymbol{y}$ by an undirected path of length $2, x v_{e}$ and $v_{e} y$ 
(where $v_{e}$ is a new vertex) with colors blue and red, respectively. Moreover, it is proved in [9] that deciding whether a 2-edge-colored graph contains a PEC $s$ - $t$ path passing through a vertex $z$ is $\mathbf{N P}$-complete. On the other side, many problems of this kind become polynomial in 2-edge-colored complete graphs. For instance, in [9] the authors proved that finding a PEC $s$ - $t$ path passing through a vertex $z$ (if any) can be solved in polynomial time. The authors of [11] produced a nice characterization of $c$-edge-colored complete graphs which admit a PEC Hamiltonian path (with a non specified source and destination), and then they deduce a new polynomial algorithm for finding it (if one exists). In [16], the authors show that generalizations of these last 2 problems are polynomial if we are restricted to $c$-edge-colored graphs with no PEC closed trails. Finally, in [20] a characterization of $c$-edge-colored multigraphs which contain a PEC Eulerian trail is given. A $O\left(n^{2} \log n\right)$ algorithm for finding a PEC Eulerian trail in $c$-edge-colored multigraphs (if one exists) is described in [7].

In [15], the authors consider edge-colored $s$ - $t$ paths, trails and walks with minimum reload costs. In this case, we are given a $c$-edge-colored graph and a $c \times c$ matrix $R=\left[r_{i, j}\right]$ (for $i, j \in I_{c}$ ) whose entries define the reload cost when changing color $i$ for color $j$. Given a trail (path) $\rho=\left(v_{1}, e_{1}, v_{2}, e_{2}, \ldots, e_{k}, v_{k+1}\right)$ between vertices $s$ and $t$, we define the reload cost of $\rho$ as $r(\rho)=\sum_{j=1}^{k-1} r_{c\left(e_{j}\right), c\left(e_{j+1}\right)}$. In [2], the authors deal with the determination of minimum directed $s$ - $t$ trails with reload costs in edge-colored digraphs whose total cost is given by the sum of reload costs (between successive colors in a trail) and positive costs associated to each arc. As discussed in [15], reload $s$ - $t$ trails (or paths) in edge-colored graphs may be converted into PEC trails (or paths) by conveniently choosing reload costs between each pair of colors (for instance, by setting $r_{i, i}=1$ and $r_{i, j}=r_{j, i}=0, \forall i, j \in I_{c}, i \neq j$. In this case, we seek a $s$ - $t$ trail (or $s$ - $t$ path) with reload cost 0$)$.

Finally, if we deal with $c$-arc-colored digraphs, the existing results concerning the complexity of finding PAC $s$ - $t$ paths and circuits are rather rare and less optimistic. To our best knowledge, there is only one result which says that deciding whether a PAC circuit exists in 2-arc-colored digraphs, is NP-complete [17].

\subsection{Contributions}

In Section 2, we deal with the problem of finding PAC $s$ - $t$ trails in $c$-arc-colored digraphs $D^{c}$ with $c \geq 2$. We show that the problem of maximizing the number of arc disjoint PAC $s-t$ trails can be solved in polynomial time. As a consequence, we prove that is polynomial to decide whether $D^{c}$ contains a directed PAC closed trail. In Section 3, we restrict our attention to path problems over $c$-arc-colored digraphs with no PAC circuits. We show that the determination of one PAC $s$ - $t$ path is NP-complete even if $D^{c}$ is a planar $c$-arc-colored digraph containing no PAC circuits and $c=\Omega\left(\left|V\left(D^{c}\right)\right|\right)$. Finally, in Section 4 we focus on $c$-arc-colored tournaments. We prove that deciding whether a $c$-arc-colored tournament $T^{c}$ (for $c=\Omega\left(\left|V\left(T^{c}\right)\right|^{2}\right)$ ) contains a PAC circuit passing through a given vertex $x$ is NP-complete. This solves a weak version of an open problem initially posed by Gutin, Sudako and Yeo [17], whose objective is to determine whether $T^{c}$ (for 
$c=2$ ) contains a PAC circuit. In addition, we prove that deciding whether $T^{c}$ has a PAC $s$ - $t$ path or a PAC Hamiltonian $s$ - $t$ path is NP-complete. Notice that there is no evident link between deciding whether a $c$-arc-colored tournament possesses a PAC $s$ - $t$ path and a PAC Hamiltonian $s$ - $t$ path, although finding a PAC $s$ - $t$ path seems to be an easier problem than finding a PAC Hamiltonian $s$ - $t$ path.

\section{$2 \quad$ PAC trails and closed trails in arc-colored digraphs}

Here, we are interested in the complexity of finding PAC $s$ - $t$ trails and closed trails in arc-colored digraphs. These problems turn out to be polynomial using minimum cost flow computation. The details of proofs are omitted due to space limitation.

Theorem 1. Given an arbitrary c-arc-colored digraph $D^{c}$, finding a PAC $s$-t trail in $D^{c}$ (if any) can be done within polynomial time.

As a consequence, we can prove the following results:

Corollary 1. Let $D^{c}$ be a c-arc-colored digraph with $c \geq 2$. The problem of finding a directed PAC closed trail in $D^{c}$ (if any) can be solved in polynomial time.

Corollary 2. The problem of maximizing the number of arc disjoint PAC $s$-t trails in $D^{c}$ can be solved in polynomial time.

\section{PAC paths in arc-colored digraphs with no PAC circuits}

Finding PEC paths, PEC trails or PEC cycles in edge-colored graphs is polynomial $[23,1]$. However finding PAC paths or PAC circuits in arc-colored digraphs seems harder. For example, the authors of [17] proved that deciding whether a 2-arccolored digraph contains a PAC circuit is NP-complete. However, the PAC $s$ - $t$ path problem is polynomial in the following simple case:

Theorem 2. If $D^{c}$ is a c-arc-colored digraph containing no circuits at all (PAC or not) and $s, t$ are two vertices of $D^{c}$ then deciding the existence of a PAC path from $s$ to $t$ is polynomial time solvable.

Unfortunately, this result does not hold in 2-arc-colored digraphs with no PAC circuits (note that non PAC circuits are allowed in this case).

Theorem 3. Deciding whether a 2-arc-colored digraph with no PAC circuits contains a PAC path from $s$ to $t$ is $\mathbf{N P}$-complete. 
Proof. We use a reduction from the Path with Forbidden Pairs Problem (PFPP, in short). In PFPP, we are given a (non-colored) digraph $D=(V, A)$, two vertices $v, w \in V$ and a collection $C=\left\{\left\{a_{1}, b_{1}\right\}, \ldots,\left\{a_{q}, b_{q}\right\}\right\}$ of pairs of vertices $\left(a_{i} \neq b_{i}\right)$ from $V \backslash\{v, w\}$. The objective is to determine whether there exists a directed path connecting $v$ to $w$ and passing through at most one vertex from each pair. This problem was shown NP-complete [12] even if $D$ is acyclic and all pairs of $C$ are required to be disjoint, i.e., $\left\{a_{i}, b_{i}\right\} \cap\left\{a_{j}, b_{j}\right\}=\emptyset$ for $i \neq j$ (see problem [GT54] page 203 in [14]).

Let $D=(V, A)$ be an acyclic digraph containing $v, w \in V$ and a subset $C$ of disjoint pairs of vertices. W.l.o.g., assume that $d_{D}^{-}(v)=d_{D}^{+}(w)=0$. The construction of $D^{c}$ is done in two steps. We build a digraph $D^{\prime}$ at first and then we build $D^{c}$ from $D^{\prime}$. The digraph $D^{\prime}=\left(V^{\prime}, A^{\prime}\right)$ is such that $V^{\prime}=V \cup\{s\}$, $A^{\prime}=A \cup A_{1}^{\prime} \cup A_{2}^{\prime}$ and vertices $v$ and $w$ are replaced by $u$ and $t$ respectively. Let $A_{1}^{\prime}:=\left\{s \boldsymbol{a}_{\mathbf{1}}, \boldsymbol{s} \boldsymbol{b}_{\mathbf{1}}, \boldsymbol{a}_{\boldsymbol{q}} \boldsymbol{u}, \boldsymbol{b}_{\boldsymbol{q}} \boldsymbol{u}\right\}$ and $A_{2}^{\prime}:=\left\{\boldsymbol{a}_{\boldsymbol{i}} \boldsymbol{a}_{\boldsymbol{i}+\mathbf{1}}, \boldsymbol{a}_{\boldsymbol{i}} \boldsymbol{b}_{\boldsymbol{i}+\mathbf{1}}, \boldsymbol{b}_{\boldsymbol{i}} \boldsymbol{a}_{\boldsymbol{i}+\mathbf{1}}, \boldsymbol{b}_{\boldsymbol{i}} \boldsymbol{b}_{\boldsymbol{i}+\mathbf{1}}: i=\right.$ $1, \ldots, q-1\}$. For the moment, notice that two arcs connecting the same pair of vertices may exist.

We build $D^{c}$ as follows: for $\operatorname{arcs}$ in $A_{1}^{\prime}, \boldsymbol{s} \boldsymbol{a}_{\mathbf{1}}$ and $\boldsymbol{s} \boldsymbol{b}_{\mathbf{1}}$ are colored in blue (color 2 ), while $\operatorname{arcs} \boldsymbol{a}_{\boldsymbol{q}} \boldsymbol{u}$ and $\boldsymbol{b}_{\boldsymbol{q}} \boldsymbol{u}$ are colored in red (color 1). Next, we apply a directed version of Häggkvist's transformation (see Subsection 1.1): each $\operatorname{arc} e=\boldsymbol{x} \boldsymbol{y}$ of $A \cup A_{2}^{\prime}$ is replaced by a directed path of length two, that is $\boldsymbol{x} \boldsymbol{v}_{\boldsymbol{e}}, \boldsymbol{v}_{\boldsymbol{e}} \boldsymbol{y}$, except for arcs incident to $t$. If $e=\boldsymbol{x} \boldsymbol{y} \in A$, then $\boldsymbol{x} \boldsymbol{v}_{\boldsymbol{e}}$ is colored in blue and $\boldsymbol{v}_{\boldsymbol{e}} \boldsymbol{y}$ is colored in red. If $e=\boldsymbol{x} \boldsymbol{t}$, then $e$ is colored in blue. By extension, $\operatorname{arcs} \boldsymbol{x} \boldsymbol{v}_{\boldsymbol{e}}, \boldsymbol{v}_{\boldsymbol{e}} \boldsymbol{y}$ are in $A$. If $e=\boldsymbol{x} \boldsymbol{y} \in A_{2}^{\prime}$ then $\boldsymbol{x} \boldsymbol{v}_{\boldsymbol{e}}$ is colored in red and $\boldsymbol{v}_{\boldsymbol{e}} \boldsymbol{y}$ is colored in blue. By extension, arcs $\boldsymbol{x} \boldsymbol{v}_{\boldsymbol{e}}, \boldsymbol{v}_{\boldsymbol{e}} \boldsymbol{y}$ are in this case in $A_{2}^{\prime}$. The construction is completed (an example is given in Figure 1).

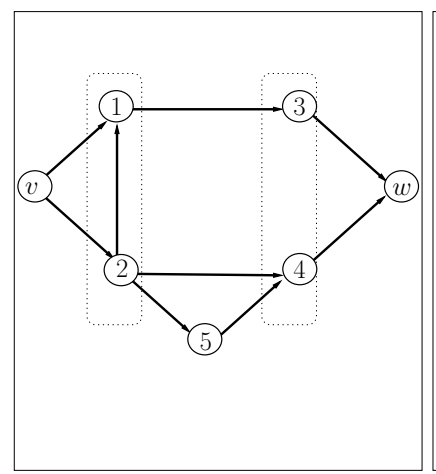

(a)

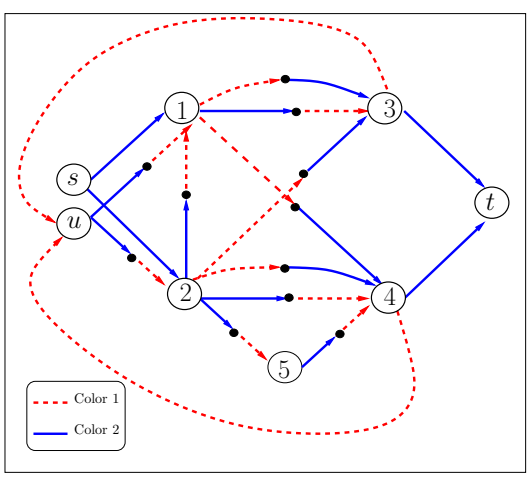

(b)

Fig. 1. Reduction from the PFPP with $C=\{\{1,2\},\{3,4\}\}$ to the PAC $s$ - $t$ path problem. Color 1 (resp., 2) corresponds to red (resp., blue).

This construction is clearly done within polynomial time and $D^{c}$ is a 2-arccolored digraph. We now give an intermediate property. 
Property 1. Any PAC path of $D^{c}$ cannot use two consecutive arcs $\boldsymbol{x} \boldsymbol{y}$ and $\boldsymbol{y} \boldsymbol{z}$ such that $\boldsymbol{x} \boldsymbol{y} \in A$ (resp., $\boldsymbol{x} \boldsymbol{y} \in A_{1}^{\prime} \cup A_{2}^{\prime}$ ) and $\boldsymbol{y} \boldsymbol{z} \in A_{1}^{\prime} \cup A_{2}^{\prime}$ (resp., $\boldsymbol{x} \boldsymbol{y} \in A$ ) except if $y=u$.

Proof. By inspection. If $\boldsymbol{x} \boldsymbol{y} \in A$ (resp., $\boldsymbol{x} \boldsymbol{y} \in A_{2}^{\prime}$ ) and $\boldsymbol{y} \boldsymbol{z} \in A_{2}^{\prime}$ (resp., $\boldsymbol{y} \boldsymbol{z} \in A$ ) then $\boldsymbol{x} \boldsymbol{y}=\boldsymbol{v}_{\boldsymbol{e}_{1}} \boldsymbol{y}$ is red (resp., blue) and $\boldsymbol{y} \boldsymbol{z}=\boldsymbol{y} \boldsymbol{v}_{\boldsymbol{e}_{\mathbf{2}}}$ is red (resp., blue). If $y \neq u$, then either $\boldsymbol{x} \boldsymbol{y}=\boldsymbol{s} \boldsymbol{a}_{\mathbf{1}}$ (resp., $\boldsymbol{x} \boldsymbol{y}=\boldsymbol{s}_{\boldsymbol{1}}$ ) (recall that $d_{D}^{-}(s)=0$ ) and $\boldsymbol{y} \boldsymbol{z} \in A$ or $\boldsymbol{x} \boldsymbol{y}=\boldsymbol{v}_{e_{1}} \boldsymbol{a}_{\boldsymbol{q}}$ (resp., $\boldsymbol{x} \boldsymbol{y}=\boldsymbol{v}_{e_{2}} \boldsymbol{b}_{q}$ ) and $\boldsymbol{y} \boldsymbol{z}=\boldsymbol{a}_{q} \boldsymbol{u}$ (resp., $\boldsymbol{y} \boldsymbol{z}=\boldsymbol{b}_{\boldsymbol{q}} \boldsymbol{u}$ ). In the first case, these two arcs $\boldsymbol{x} \boldsymbol{y}$ and $\boldsymbol{y} \boldsymbol{z}$ are blue, while in the second case, these two arcs $\boldsymbol{x} \boldsymbol{y}$ and $\boldsymbol{y} \boldsymbol{z}$ are red.

From Property 1, we deduce that any PAC path of $D^{c}$ from $s$ to $t$ uses some arcs in $A_{1}^{\prime} \cup A_{2}^{\prime}$ at first and after it uses some arcs in $A$ (after passing through $u)$.

Let us show that $D^{c}$ does not contain any PAC circuit. Since $\left(V^{\prime}, A\right)$ has no circuits (by hypothesis) and $\left(V^{\prime}, A_{1}^{\prime} \cup A_{2}^{\prime}\right)$ has no circuits (by construction), any circuit of $D^{c}$ must contain two consecutive arcs such that the first arc is in $A$ (resp., $A_{1}^{\prime} \cup A_{2}^{\prime}$ ) and the second arc is in $A_{1}^{\prime} \cup A_{2}^{\prime}$ (resp., A). Using Property 1, the circuit is not PAC.

Finally, using Property 1, we claim that we have a directed path from $v$ to $w$ in $D$ and visiting at most one vertex from each pair of $C$, if and only if, we have a PAC path from $s$ to $t$ in $D^{c}$. To see that, let $\Gamma$ with $|\Gamma| \leq q$ be a subset of vertices of $C$ belonging to a directed path from $v$ to $w$ in $D$. As a consequence, we can construct a directed PAC sub-path from $u$ to $t$ in $D^{c}$, say $\alpha$, visiting the same set $\Gamma$ of vertices and only containing $\operatorname{arcs}$ of $A$. Therefore, from Property 1 , we can determine a PAC path from $s$ to $t$ in $D^{c}$ by concatenating a PAC sub-path from $s$ to $u$ and containing no vertices of $\Gamma$ (only with $\operatorname{arcs}$ of $A_{1}^{\prime} \cup A_{2}^{\prime}$ ), which always exist in this case, with the PAC sub-path $\alpha$ from $u$ to $t$. Conversely, consider a PAC path from $s$ to $t$ in $D^{c}$ (note that all PAC $s$ - $t$ paths in $D^{c}$ contain vertex $u$ ). Thus, by Property 1, it follows that the associated PAC sub-path from $s$ to $u$ only contains arcs of $A_{1}^{\prime} \cup A_{2}^{\prime}$ and the PAC sub-path from $u$ to $t$ only contains $\operatorname{arcs}$ of $A$ (each of them containing at most one vertex of $\left\{a_{i}, b_{i}\right\}$ for $i=1, . ., q$ ). After deleting all arcs of $A_{1}^{\prime} \cup A_{2}^{\prime}$ in $D^{c}$, the resulting path from $u$ to $t$ will be directely associated to a path from $v$ to $w$ in $D$ passing through at most one vertex from each pair of $C$.

Now, we show that our previous theorem can be extended to include any number of colors. Formally, we have the following result:

Corollary 3. Deciding whether a c-arc-colored digraph $D^{c}$ with no PAC circuits contains a PAC $s$-t path is $\mathbf{N P}$-complete, even if $c=\Omega\left(\left|V\left(D^{c}\right)\right|^{2}\right)$.

Theorem 3 can also be extended to planar $c$-arc-colored digraphs.

Corollary 4. Deciding whether a planar c-arc-colored digraph $D_{P}^{c}$ with no PAC circuits contains a PAC $s$-t path is $\mathbf{N P}$-complete even for $c=\Omega\left(\left|V\left(D_{P}^{c}\right)\right|\right)$. 


\section{PAC circuits, paths and Hamiltonian paths in $c$-arc-colored tournaments}

A tournament is a digraph which corresponds to a complete asymmetric binary relation. As indicated previously, one can build a tournament as follows: take a complete undirected graph and assign a direction to each edge. The problems of finding PAC $s$ - $t$ paths and PAC circuits in $c$-arc-colored tournaments are challenging. For example, the complexity of determining a PAC circuit in a 2-arc-colored tournament is posed in $[17,5]$. Here, we propose and solve a weaker version of this problem, we show that deciding whether a $c$-arc-colored tournament contains a PAC circuit passing through a given vertex $x$ is NP-complete. As a consequence, we prove that finding PAC $s$ - $t$ paths in tournaments is also NP-complete.

We also deal with the determination of PAC Hamiltonian $s$ - $t$ paths in arccolored tournaments. When restricted to uncolored tournaments, one of the earliest results is Rédei's theorem, which states that every tournament has an Hamiltonian directed path (the endpoints are not specified). More recently, in [6] the authors gave a polynomial algorithm to find an Hamiltonian directed $s$ - $t$ path (if one exists) in an uncolored tournament. Recently in $[11,5]$ (using a nice characterization) the authors show that the problem of finding PEC Hamiltonian $s$ - $t$ path is polynomial in $c$-edge-colored complete graphs for $c \geq 3$, solving a conjecture posed in [4] (the case $c=2$ was previously solved in [4]). Unfortunately, we prove that these results cannot be extended to the directed case.

Thus, we begin with the following result:

Theorem 4. Deciding whether a c-arc-colored tournament contains a PAC circuit visiting a given vertex $x$ is $\mathbf{N P}$-complete even for $c=\Omega\left(\left|V\left(D^{c}\right)\right|\right)$.

Proof. Here, we only deal with $c=2$ since our proof can be easily extended for $c=\Omega\left(\left|V\left(D^{c}\right)\right|\right)$ (see the Appendix for details). Thus, we start from the 2-arccolored digraph $D^{c}=\left(V^{\prime}, A^{\prime}\right)$ built in Theorem 3 and we complete it in order to get a tournament $T^{c}$. The idea is to get a tournament whose PAC circuits passing through $x=s$ (if one exists) also visit vertex $t$. Then, directed paths from $v$ to $w$ in $D$ (visiting at most one vertex from each pair of $C$ ), instance of the Path with Forbidden Pairs Problem, correspond to PAC circuits passing through $s$ in $T^{c}$ and vice-versa.

Recall that in the construction of $D^{c}$ (see the proof of Theorem 3), we replace each $\operatorname{arc} e \in A$ (resp., $e$ from $A_{2}^{\prime}$ ), except those which are incident to $t$, by a directed path of length two in $A$ (resp., in $A_{2}^{\prime}$ ) where the added vertex is denoted by $v_{e}$. If $e \in A$ (resp. $e \in A_{2}^{\prime}$ ) then we suppose that $v_{e} \in V(A)$ (resp., $\left.v_{e} \in V\left(A_{2}^{\prime}\right)\right)$.

Now, we show how to build the tournament $T^{c}$. The construction is done in four steps:

(1) Build a set of $\operatorname{arcs} A_{3}^{\prime}$ as follows. Add a red arc $\boldsymbol{t} \boldsymbol{s}$ and a blue arc $\boldsymbol{u} \boldsymbol{s}$. Do $A\left(D^{c}\right) \leftarrow A\left(D^{c}\right) \cup A_{3}^{\prime}$. Then, add a blue arc $\boldsymbol{t} \boldsymbol{x}$ for each $x \notin N_{D^{c}}(t)$, a blue arc $\boldsymbol{x} \boldsymbol{u}$ for each $x \notin N_{D^{c}}(u)$ and a blue arc $\boldsymbol{x s}$ for each $x \notin N_{D^{c}}(s)$. Do $A\left(D^{c}\right) \leftarrow A\left(D^{c}\right) \cup A_{3}^{\prime}$. 
(2) Build a set of arcs $A_{4}^{\prime}$ as follows. Choose an arbitrary vertex $v_{e}$ of $V(A)$ (resp., $V\left(A_{2}^{\prime}\right)$ ) with an incoming blue (resp., red) arc $\boldsymbol{y} \boldsymbol{v}_{\boldsymbol{e}}$ (resp., $\boldsymbol{a}_{\boldsymbol{i}} \boldsymbol{v}_{\boldsymbol{e}}$ or $\boldsymbol{b}_{\boldsymbol{i}} \boldsymbol{v}_{\boldsymbol{e}}$ ), and add a blue (resp., red) arc $\boldsymbol{v}_{\boldsymbol{e}} \boldsymbol{x}$ for every $x \notin N_{D^{c}}\left(v_{e}\right)$. Let $A_{4}^{\prime}$ be this new set of arcs and do $A\left(D^{c}\right) \leftarrow A\left(D^{c}\right) \cup A_{4}^{\prime}$. Repeat the process for the remaining vertices $v_{e}$ of $V(A)$ (resp., $V\left(A_{2}^{\prime}\right)$ ) by following an arbitrary order.

(3) Build a set of blue $\operatorname{arcs} A_{5}^{\prime}=\left\{\boldsymbol{a}_{\boldsymbol{q}} \boldsymbol{x}: \forall x \notin N_{D^{c}}\left(a_{q}\right)\right\} \cup\left\{\boldsymbol{b}_{\boldsymbol{q}} \boldsymbol{y}: \forall y \notin\left(N_{D^{c}}\left(b_{q}\right) \cup\right.\right.$ $\left.\left.\left\{a_{q}\right\}\right)\right\}$. Recall that $\left\{a_{q}, b_{q}\right\}$ is the last pair of $C$. Set $A\left(D^{c}\right) \leftarrow A\left(D^{c}\right) \cup A_{5}^{\prime}$.

(4) Build a set $A_{6}^{\prime}$ of blue arcs with endpoints in $V\left(D^{c}\right) \backslash\left(\left\{s, u, t, a_{q}, b_{q}\right\} \cup\left\{v_{e}\right.\right.$ : $\left.\left.v_{e} \in V(A) \cup V\left(A_{2}^{\prime}\right)\right\}\right)$ and arbitrary directions. Set $A\left(D^{c}\right) \leftarrow A\left(D^{c}\right) \cup A_{6}^{\prime}$.

The construction is completed. It is clearly done within polynomial time, and $T^{c}$ is a 2-arc-colored tournament. We now give some useful properties:

Property 2. The following properties hold:

(i) Any PAC circuit passing through $s$ (resp., $u$ ) in $T^{c}$ uses $\boldsymbol{t} \boldsymbol{s}$ and one arc among $\left\{\boldsymbol{s} \boldsymbol{a}_{1}, \boldsymbol{s} \boldsymbol{b}_{\boldsymbol{1}}\right\}$ (resp., uses exactly one arc among $\left\{\boldsymbol{a}_{\boldsymbol{q}} \boldsymbol{u}, \boldsymbol{b}_{\boldsymbol{q}} \boldsymbol{u}\right\}$ and one $\left.\operatorname{arc} \boldsymbol{u} \boldsymbol{v}_{\boldsymbol{e}} \in A\right)$.

(ii) No PAC circuit passing through $s$ in $T^{c}$ uses an arc of $A_{4}^{\prime}$.

(iii) No PAC circuit passing through $s$ in $T^{c}$ uses an $\operatorname{arc}$ of $A_{5}^{\prime} \cup A_{6}^{\prime}$.

Proof. For $(i)$. Due to step (1) of the above procedure, there is a unique red arc incident to $s$ (resp., $t$ ) which is $\boldsymbol{t} \boldsymbol{s}$. Thus, any PAC circuit passing through $s$ also visits $t$. Moreover, vertex $s$ only has two outgoing $\operatorname{arcs} \boldsymbol{x} \boldsymbol{a}_{\mathbf{1}}$ and $\boldsymbol{x} \boldsymbol{b}_{\mathbf{1}}$ which are colored in blue.

Concerning vertex $u, \boldsymbol{a}_{\boldsymbol{q}} \boldsymbol{u}$ and $\boldsymbol{b}_{\boldsymbol{q}}$ are the only red arcs incident to $u$. Thus, if a PAC circuit visits $u$ then it contains one of these two arcs as incoming arc and one arc $\boldsymbol{u} \boldsymbol{v}_{\boldsymbol{e}} \in A$ as outgoing arc. Actually, vertex $u$ has only $\operatorname{arcs} \boldsymbol{u} \boldsymbol{v}_{\boldsymbol{e}} \in A$ and $\boldsymbol{u} \boldsymbol{s}$ as outgoing arcs and no PAC circuit can use the blue arc $\boldsymbol{u} \boldsymbol{s}$ since all arcs going out of $s$ are blue.

For (ii). By contradiction, assume that there is a PAC circuit passing through $s, \rho=\left(v_{1}, e_{1}, \ldots, e_{k}, v_{k+1}\right)$ with $v_{1}=v_{k+1}=s$ and containing some arcs of $A_{4}^{\prime}$. Consider the first $\operatorname{arc} e_{p} \in A_{4}^{\prime}$ used by $\rho$ (i.e., $e_{q} \notin A_{4}^{\prime}$ for $q=1, \ldots, p-1$ ). By construction $e_{p}=\boldsymbol{v}_{\boldsymbol{e}} \boldsymbol{x}$ and from (i), we deduce $k>p>1$ (i.e., $x \notin\{s, t\}$ ). Since $e_{p-1} \notin A_{4}^{\prime}$ and $e_{p-1} \notin A_{3}^{\prime}$ from $(i)$, arc $e_{p-1}=\boldsymbol{y} \boldsymbol{v}_{\boldsymbol{e}} \in A \cup A_{2}^{\prime}$. Thus, $e_{p-1}$ has the same color as $e_{p}$, which is a contradiction.

For (iii). By contradiction. Firstly assume that there is a PAC circuit passing through $s, \rho=\left(v_{1}, e_{1}, \ldots, e_{k}, v_{k+1}\right)$ with $v_{1}=v_{k+1}=s$ and containing some $\operatorname{arcs}$ of $A_{5}^{\prime}$. Like previously, consider the first $\operatorname{arc} e_{p} \in A_{5}^{\prime}$ of $\rho$ (i.e., $e_{q} \notin A_{5}^{\prime}$ for $q=1, \ldots, p-1$ ). W.l.o.g., suppose that $e_{p}=\boldsymbol{a}_{\boldsymbol{q}} \boldsymbol{x}$ (the same result holds for $\left.e_{p}=\boldsymbol{b}_{\boldsymbol{q}} \boldsymbol{x}\right)$; we get $x \neq u$ from $(i)$. Then, $e_{p-1}=\boldsymbol{v}_{\boldsymbol{e}} \boldsymbol{a}_{\boldsymbol{q}} \in A$ is colored in red and from (ii) we deduce that $e_{p-2}=\boldsymbol{y} \boldsymbol{v}_{\boldsymbol{e}} \in A$ and is colored in blue. Since all arcs in $A_{6}^{\prime}$ are blue like $e_{p-2}$, by induction we deduce that $e_{q} \in A$ for $q=1, \ldots, p-1$. We obtain a contradiction since from $(i) e_{1} \in A_{1}^{\prime}$ (i.e., $e_{1} \in\left\{\boldsymbol{s} \boldsymbol{a}_{1}, \boldsymbol{s} \boldsymbol{b}_{\mathbf{1}}\right\}$ ).

Now, suppose that a PAC circuit passing through $s, \rho=\left(v_{1}, e_{1}, \ldots, e_{k}, v_{k+1}\right)$ with $v_{1}=v_{k+1}=s$ contains some arcs in $A_{6}^{\prime}$. Consider the last arc $e_{p}=\boldsymbol{x} \boldsymbol{y} \in A_{6}^{\prime}$ used 
by $\rho$ (i.e., $e_{q} \notin A_{6}^{\prime}$ for $q=p+1, \ldots, k+1$ ). Since $e_{p}$ is colored in blue and $y \neq t$ (from $(i)$ ), we deduce that $e_{p+1}$ is colored in red. Then, we get $y=a_{i}$ or $y=b_{i}$ and $e_{p+1}=\boldsymbol{y} \boldsymbol{v}_{\boldsymbol{e}} \in A_{2}^{\prime}$ since $e_{p+1} \notin A_{6}^{\prime}$. Moreover, from (ii), $e_{p+2}=\boldsymbol{v}_{\boldsymbol{e}} \boldsymbol{z} \in A_{2}^{\prime}$ is colored in blue. Now, since $e_{k} \in A$ (the arc of $\rho$ incoming in vertex $t$ ) is also colored in blue, the PAC subpath of $\rho$ from $x$ to $t=v_{k}$ must contain arc $\boldsymbol{a}_{\boldsymbol{q}} \boldsymbol{u}$ or $\boldsymbol{b}_{\boldsymbol{q}} \boldsymbol{u}$ (using Property 1 of Theorem 3, it is the only way to flip $\operatorname{arcs}$ of $A_{2}^{\prime}$ to $\operatorname{arcs}$ of $A$ ). Thus, this PAC circuit $\rho$ can be decomposed into three PAC paths: $\rho_{1}$ from $y$ to $u, \rho_{2}$ from $u$ to $s$ (and containing arc $e_{k+1}=\boldsymbol{t s}$ ) and $\rho_{3}$ from $s$ to $y$. In particular, the PAC path $\rho_{3}$ begins with a blue arc (by $(i)$ ), only uses arcs in $A_{2}^{\prime}$ and ends by a blue arc, which is impossible since $\rho_{3}$ does not contain $u$. Actually, path $\rho_{3}$ cannot use some arcs of $A_{6}^{\prime}$. We have $e_{2}=\boldsymbol{x}_{\mathbf{1}} \boldsymbol{v}_{\boldsymbol{e}} \in A_{2}^{\prime}$ with $x_{1} \in\left\{a_{1}, b_{1}\right\}$ (since the arc must be colored in red) and using (ii), arc $e_{3}=\boldsymbol{v}_{e} \boldsymbol{x}_{\mathbf{2}}$ with $x_{2} \in\left\{a_{2}, b_{2}\right\}$ is colored in blue. Thus, $e_{4} \notin A_{5}^{\prime} \cup A_{6}^{\prime}$. Then, the result follows by induction. Notice that it may exist a PAC circuit containing one arc $e=\boldsymbol{x} \boldsymbol{y} \in A_{6}^{\prime}$ (but not passing through $s$ ). In this case, this PAC circuit is composed of two PAC paths $\rho_{1}$ from $y$ to $u$ and $\rho_{2}$ from $u$ to $y$ : $\rho_{1}$ only uses arcs of $A_{2}^{\prime}$ from $y$ to $a_{q}$ (or $b_{q}$ ) and uses arc $\boldsymbol{a}_{\boldsymbol{q}} \boldsymbol{u} \in A_{1}^{\prime}$ (or $\boldsymbol{b}_{\boldsymbol{q}} \boldsymbol{u} \in A_{1}^{\prime}$ ) while $\rho_{2}$ only uses $\operatorname{arcs}$ of $A$ from $u$ to $x$ and uses arc $e=\boldsymbol{x} \boldsymbol{y} \in A_{6}^{\prime}$.

Using Properties 1 and 2, we can easily see that we have a directed path from $u$ to $w$ in $D$ and visiting at most one vertex from each pair of $C$, if and only if, we have a PAC circuit passing through $s$ in $T^{c}$.

Corollary 5. Deciding whether a c-arc-colored tournament $T^{c}$ contains a PAC $s$-t path is $\mathbf{N P}$-complete even for $c=\Omega\left(\left|V\left(T^{c}\right)\right|^{2}\right)$.

Proof. In the proof of Theorem 4, we have a PAC circuit passing through $s$ if and only if we have a PAC $s$ - $t$ path in $T^{c}$.

We finish the paper by considering the PAC Hamiltonian $s$ - $t$ path problem.

Theorem 5. Deciding whether a 2-arc-colored tournament $T^{c}$ contains a PAC Hamiltonian s-t path is $\mathbf{N P}$-complete.

Proof. We use a reduction from the directed Hamiltonian $s^{\prime}-t^{\prime}$ path problem in general uncolored digraphs (DHPP in short). Given a digraph $D=(V, A)$ and two vertices $s^{\prime}, t^{\prime}$, DHPP asks whether a directed Hamiltonian $s^{\prime}-t^{\prime}$ path exists. DHPP is NP-complete (see problem [GT39] page 199 in [14]).

Let $D=(V, A)$ be a digraph where $V=\left\{v^{1}, \ldots, v^{n}\right\}$ and $v^{1}=s^{\prime}, v^{n}=t^{\prime}$, instance of DHPP. W.l.o.g., assume that $d_{D}^{-}\left(v^{1}\right)=d_{D}^{+}\left(v^{n}\right)=0$. The construction of the 2-arc-colored tournament $T^{c}$ is done in two steps: we first build a 2-arccolored digraph $D^{c}$ and then we complete $D^{c}$ into $T^{c}$.

The 2-arc-colored digraph $D^{c}=\left(V^{\prime}, A^{\prime}\right)$ is built in the following way: $V^{\prime}=$ $\left\{v_{\text {in }}^{i}, v_{\text {out }}^{i}: i=1, \ldots, n\right\}$ and $A^{\prime}=A_{1}^{\prime} \cup A_{2}^{\prime}$ where $A_{1}^{\prime}=\left\{\boldsymbol{v}_{\text {out }}^{i} \boldsymbol{v}_{\boldsymbol{i n}}^{j}: \boldsymbol{v}^{i} \boldsymbol{v}^{j} \in A\right\}$ and $A_{2}^{\prime}=\left\{\boldsymbol{v}_{\boldsymbol{i n}}^{i} \boldsymbol{v}_{\text {out }}^{i}: i=1, \ldots, n\right\}$. Arcs in $A_{1}^{\prime}$ are colored in red while arcs in $A_{2}^{\prime}$ are colored in blue. See Figure 2 for an illustration of $D^{c}$.

Next we build the tournament $T^{c}$ from $D^{c}$ as follows. For every missing arc in $D^{c}$, we apply the following procedure where $1 \leq i<j \leq n$ is assumed. If 

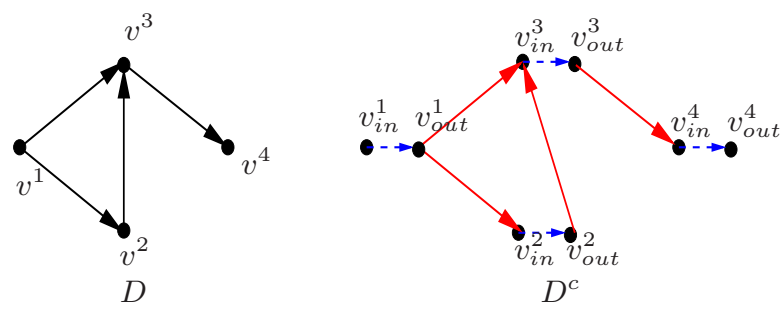

Fig. 2. A digraph $D$ and the 2-arc-colored digraph $D^{c}$. Dotted arcs are colored in blue and rigid arcs are colored in red.

the endpoints of the missing arc are $v_{i n}^{i}$ and $v_{i n}^{j}$ (resp., $v_{i n}^{i}$ and $v_{\text {out }}^{j}$ ), add a blue $\operatorname{arc} \boldsymbol{v}_{\boldsymbol{i n}}^{j} \boldsymbol{v}_{\boldsymbol{i n}}^{\boldsymbol{i}}$ (resp., $\boldsymbol{v}_{\boldsymbol{o u t}}^{j} \boldsymbol{v}_{\boldsymbol{i n}}^{i}$ ). If the endpoints of the missing arc are $v_{\text {out }}^{i}$ and $v_{\text {in }}^{j}$ (resp., $v_{\text {out }}^{i}$ and $v_{\text {out }}^{j}$ ), add a red $\operatorname{arc} \boldsymbol{v}_{\boldsymbol{i n}}^{j} \boldsymbol{v}_{\text {out }}^{i}\left(\operatorname{resp} ., \boldsymbol{v}_{\text {out }}^{j} \boldsymbol{v}_{\text {out }}^{i}\right)$. These new blue (resp., red) arcs define a set denoted by $A_{2}^{\prime \prime}$ (resp., $A_{1}^{\prime \prime}$ ).

The construction is completed (see Figure 3 for an illustration). It is clearly done within polynomial time. The resulting tournament is 2-arc-colored. Its blue arcs belong to $A_{2}^{\prime} \cup A_{2}^{\prime \prime}$ while its red arcs belong to $A_{1}^{\prime} \cup A_{1}^{\prime \prime}$. Let us give an intermediate property.
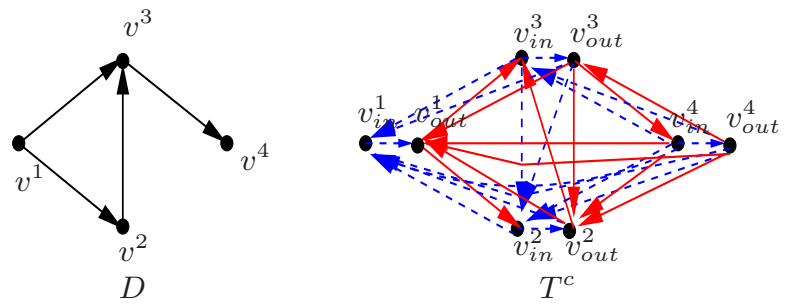

Fig. 3. A digraph $D$ and the 2-arc-colored tournament $T^{c}$. Dotted arcs are colored in blue and rigid arcs are colored in red.

Property 3. No PAC path from $v_{\text {in }}^{1}$ to $v_{\text {out }}^{n}$ in $T^{c}$ can use an arc of $A_{1}^{\prime \prime} \cup A_{2}^{\prime \prime}$.

Proof. By contradiction suppose that a PAC path $\rho=\left(v_{0}, e_{0}, v_{1}, e_{1}, \ldots\right.$, $\left.e_{k}, v_{k+1}\right)$ linking $v_{0}=v_{\text {in }}^{1}$ to $v_{k+1}=v_{\text {out }}^{n}$ uses some arcs of $A_{1}^{\prime \prime} \cup A_{2}^{\prime \prime}$. Consider the last arc $e_{p} \in A_{1}^{\prime \prime} \cup A_{2}^{\prime \prime}$ used by $\rho$ (that is $e_{q} \notin A_{1}^{\prime \prime} \cup A_{2}^{\prime \prime}$ for $q=p+1, \ldots, k+1$ ). If $e_{p}=\boldsymbol{v}_{\boldsymbol{i n}}^{\boldsymbol{j}} \boldsymbol{v}_{\boldsymbol{i n}}^{\boldsymbol{i}}$ or $e_{p}=\boldsymbol{v}_{\boldsymbol{o u t}}^{\boldsymbol{j}} \boldsymbol{v}_{\boldsymbol{i n}}^{\boldsymbol{i}}(i<j)$ then it belongs to $A_{2}^{\prime \prime}$ and it is blue. We have $v_{\text {in }}^{i} \neq v_{\text {out }}^{n}$ so the path must contain an arc going out of $v_{\text {in }}^{i}$ which does not belong to $A_{1}^{\prime \prime} \cup A_{2}^{\prime \prime}$. This $\operatorname{arc} e_{p+1}=\boldsymbol{v}_{\boldsymbol{i n}}^{\boldsymbol{i}} \boldsymbol{v}_{\text {out }}^{\boldsymbol{i}}$ is blue, contradiction. Otherwise, $e_{p}=\boldsymbol{v}_{\boldsymbol{i n}}^{\boldsymbol{j}} \boldsymbol{v}_{\text {out }}^{\boldsymbol{i}}(i \neq j)$ or $e_{p}=\boldsymbol{v}_{\text {out }}^{\boldsymbol{j}} \boldsymbol{v}_{\text {out }}^{\boldsymbol{i}}$. Therefore $e_{p} \in A_{1}^{\prime \prime}$ and it is red. We have $v_{\text {out }}^{i} \neq v_{\text {out }}^{n}$ since $\boldsymbol{v}_{\boldsymbol{i n}}^{n} \boldsymbol{v}_{\text {out }}^{n}$ is the unique arc coming into $v_{\text {out }}^{n}$. Then, the path 
must contain an $\operatorname{arc} e_{p+1} \notin A_{1}^{\prime \prime} \cup A_{2}^{\prime \prime}$ going out of $v_{\text {out }}^{i}$ but all arcs of $A_{1}^{\prime} \cup A_{2}^{\prime}$ going out of $v_{\text {out }}^{i}$ are red since they belong to $A_{1}^{\prime}$, contradiction.

We deduce from Property 3 that any PAC path from $v_{\text {in }}^{1}$ to $v_{\text {out }}^{n}$ in $T^{c}$ only uses arcs of $A_{1}^{\prime} \cup A_{2}^{\prime}$. Thus, $D$ admits a directed Hamiltonian path from $s^{\prime}=v^{1}$

to $v^{n}=t^{\prime}$, if and only if, $T^{c}$ has a PAC Hamiltonian path from $s=v_{\text {in }}^{1}$ to $t=v_{\text {out }}^{n}$.

\section{References}

1. A. Abouelaoualim, K. C. Das, L. Faria, Y. Manoussakis, C. A. Martinhon and R. Saad. Paths and trails in edge-colored graphs, Theoretical Computer Science, 409(3), 497-510, 2008.

2. E. Amaldi, G. Galbiati and F. Maffioli. On the minimum reload cost paths, tours and flows, Proc. CTW 08, 112-115, 2008.

3. J. Bang-Jensen and G. Gutin. Alternating cycles and paths in edge-coloured multigraphs: a survey, Discrete Mathematics, 165/166, 39-60, 1997.

4. J. Bang-Jensen and G. Gutin. Alternating cycles and trails in 2-edge-coloured complete multigraphs, Discrete Mathematics, 188, 61-72, 1998.

5. J. Bang-Jensen and G. Gutin. Digraphs: Theory, Algorithms and Applications, First Edition, Springer-Verlag, London Springer Monographs in Mathematics, 2006.

6. J. Bang-Jensen, Y. Manoussakis and C. Thomassen. A Polynomial Algorithm for Hamiltonian-Connectedness in Semicomplete Digraphs, J. Algorithms, 13(1), 114127, 1992.

7. A. Benkouar, Y. Manoussakis, V. Th. Paschos and R. Saad. On the complexity of Hamiltonian and Eulerian problems in edge-colored complete graphs, RAIRO Journal On Operations Research, 30(4), 417-438, 1996.

8. J. A. Bondy and U. S. R. Murty Graph Theory (Graduate Texts in Mathematics), Springer, 2008.

9. W. S. Chou, Y. Manoussakis, O. Megalakaki, M. Spyratos and Z. Tuza. Paths through fixed vertices in edge-colored graphs, Mathématiques et Sciences Humaines, 127, 49-58, 1994.

10. D. Dorniger Hamiltonian circuits determining the order of chromossomes, Discrete Applied Mathematics, 50, 159-168, 1994.

11. J. Feng, H.-E. Giesen, Y. Guo, G. Gutin, T. Jensen and A. Rafiey. Characterization of edge-colored complete graphs with properly colored Hamilton paths, J. Graph Theory, 53, 333-346, 2006.

12. H. Gabow, S. Maheshwari and L. Osterweil. On two Problems in the Generation of Program Test Paths, IEEE Trans. on Soft. Eng., SE-2, (3) 227-231, 1976.

13. I. Gamvros. Satellite network design, optimization and management PhD thesis, University of Maryland, 2006.

14. M. R. Garey and D. S. Johnson. Computers and Intractability: A Guide to the Theory of NP-Completeness, W. H. Freeman and Company, New York, 1979.

15. L. Gourvès, A. Lyra, C. Martinhon and J. Monnot. The minimum reload s-t path/trail/walk problems, In Proc. of SOFSEM'09, LNCS 5404, 621-632, 2009.

16. L. Gourvès, A. Lyra, C. Martinhon, J. Monnot and F. Protti. On s-t paths and trails in edge-colored graphs, Proc. LAGOS 09, Electronic Notes in Discrete Mathematics, 35, 221-226, 2009. 
17. G. Gutin, B. Sudakov and A. Yeo. Note on alternating directed cycles, Discrete Mathematics, 191, 101-107, 1998.

18. G. Gutin and E. J. Kim. Properly Coloured Cycles and Paths: Results and Open Problems, Golumbic Festschrift, LNCS 5420, 200-208, 2009.

19. T.C. Hu, Y.S. KuO Graph folding and programmable logical arrays, Networks, 17, 19-37, 1987.

20. A. Kotizig. Moves without forbidden transitions in a graph, Math. Fyz. Cazopis, 18, 76-80, 1968.

21. P. Pevzner. DNA physical mapping and properly edge-colored eurelian cycles in colored graphs, Algorithmica, 13, 77-105, 1995.

22. P. Pevzner. Computational Molecular Biology: An Algorithmic Approach, The MIT Press, 2000.

23. S. Szeider. Finding paths in graphs avoiding forbidden transitions, Discrete Applied Mathematics, 126, 261-273, 2003.

24. A. Yeo. A note on Alternating Cycles in Edge-coloured Graphs, Journal of Combinatorial Theory, Series B- 69, 222-225, 1997. 